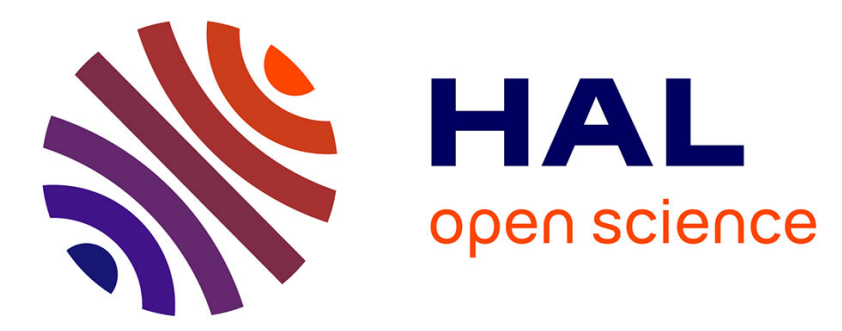

\title{
Change Detection for High Resolution Satellite Images, based on SIFT descriptors and an a Contrario approach
}

Flora Dellinger, Julie Delon, Yann Gousseau, Julien Michel, Florence Tupin

\section{To cite this version:}

Flora Dellinger, Julie Delon, Yann Gousseau, Julien Michel, Florence Tupin. Change Detection for High Resolution Satellite Images, based on SIFT descriptors and an a Contrario approach. IGARSS 2014, Jul 2014, Québec, Canada. hal-01059366

\section{HAL Id: hal-01059366 https://hal.science/hal-01059366}

Submitted on 29 Aug 2014

HAL is a multi-disciplinary open access archive for the deposit and dissemination of scientific research documents, whether they are published or not. The documents may come from teaching and research institutions in France or abroad, or from public or private research centers.
L'archive ouverte pluridisciplinaire HAL, est destinée au dépôt et à la diffusion de documents scientifiques de niveau recherche, publiés ou non, émanant des établissements d'enseignement et de recherche français ou étrangers, des laboratoires publics ou privés. 


\title{
CHANGE DETECTION FOR HIGH RESOLUTION SATELLITE IMAGES, BASED ON SIFT DESCRIPTORS AND AN A CONTRARIO APPROACH
}

\author{
Flora Dellinger ${ }^{1}$, Julie Delon ${ }^{2}$, Yann Gousseau ${ }^{1}$, Julien Michel ${ }^{3}$, Florence Tupin ${ }^{1}$ \\ ${ }^{1}$ Institut Mines-Telecom, Telecom ParisTech, LTCI CNRS, Paris, France \\ ${ }^{2}$ MAP5, Universite Paris Descartes, Paris, France \\ ${ }^{3} \mathrm{CNES}, \mathrm{DCT} / \mathrm{SI} / \mathrm{AP}$, Toulouse, France
}

\begin{abstract}
In disaster situations, remote sensing images are very useful to quickly assess damages. However, the choice of available images for the studied area is frequently limited. It is often needed to compare images acquired by different sensors and with different acquisition conditions. We propose a new feature-based approach to detect changes between a pair of either optical or radar images. This approach is based on the SIFT algorithm and an a contrario approach. It can deal with multi-resolutions, multi-sensors and multi-incidence angles situations, and it offers promising results.
\end{abstract}

Index Terms - SAR image, SIFT, change detection, local descriptors, RANSAC, a contrario methods, image comparison

\section{INTRODUCTION}

The last years have seen an increase of the number of high resolution remote sensing satellites and of situations where it is necessary to compare images from different sensors and even different modalities such as radar and optical, possibly with different resolutions and incidence angles. This is especially common for change detection in disaster situations, where the choice of available images is often limited. Besides some issues are particularly important in high resolution satellite images: increase of image complexity, occlusion and shadow effects, misregistration errors, etc. The pixel-based approaches are thus not appropriate and feature-based ones should be preferred. The Scale Invariant Feature Transform (SIFT) [1] is a widely used computer vision algorithm for the detection and matching of interest points. Its invariances to image translation, scaling, rotation and partially to illumination changes makes it suitable for these situations. In this paper, we propose an a contrario approach based on SIFT keypoints matching to identify change areas. This method does not require a pre-registration step and works on pairs of either optical or SAR images.

\section{CHANGE DETECTION FOR MULTI-SENSORS AND MULTI-RESOLUTIONS SITUATIONS}

Only few papers deal with change detection using high resolution images from different sensors and/or resolutions. Most works have focused on multi-temporal data with images from the same sensor or with low resolution images. The difficulty to compare images from different sensors (leading to radiometric, resolution and geometric issues), associated with the complexity inherent to high resolution images, turns it into a challenging problem. Some algorithms propose to use pixelbased approaches on co-registered images, while still considering issues of misregistration. The GeoCDX method [2] extracts features such as entropy, skewness and lines, and operates a pixel-based change detection with a spatial tolerance to misregistration. Bryant et al. [3] perform a background characterization from the multi-spectral bands of the image, followed by a spectral differentiation and a detection of change pixels with a Bayesian classifier. Gamba et al. [4] use a feature-based change detection technique (with edge detection and matching) to correct misdetections, due to misregistrations or miscalibrations, from an area-based change detection map.

To avoid the problems of misregistration, we propose here to use a feature-based approach with no need for prior registration and calibration. This method can be applied to a pair of high resolution optical or SAR images, possibly from different sensors and with different resolutions and/or incidence angles.

\section{PROPOSED METHODOLOGY}

First, interest points are detected in the two images and matched, with help of the SIFT and RANSAC algorithms (section 3.1). These points give informations about the areas presenting structures and the ones with changes (section 3.2). Then, the change areas are selected through an a contrario approach (section 3.3 and 3.4). 

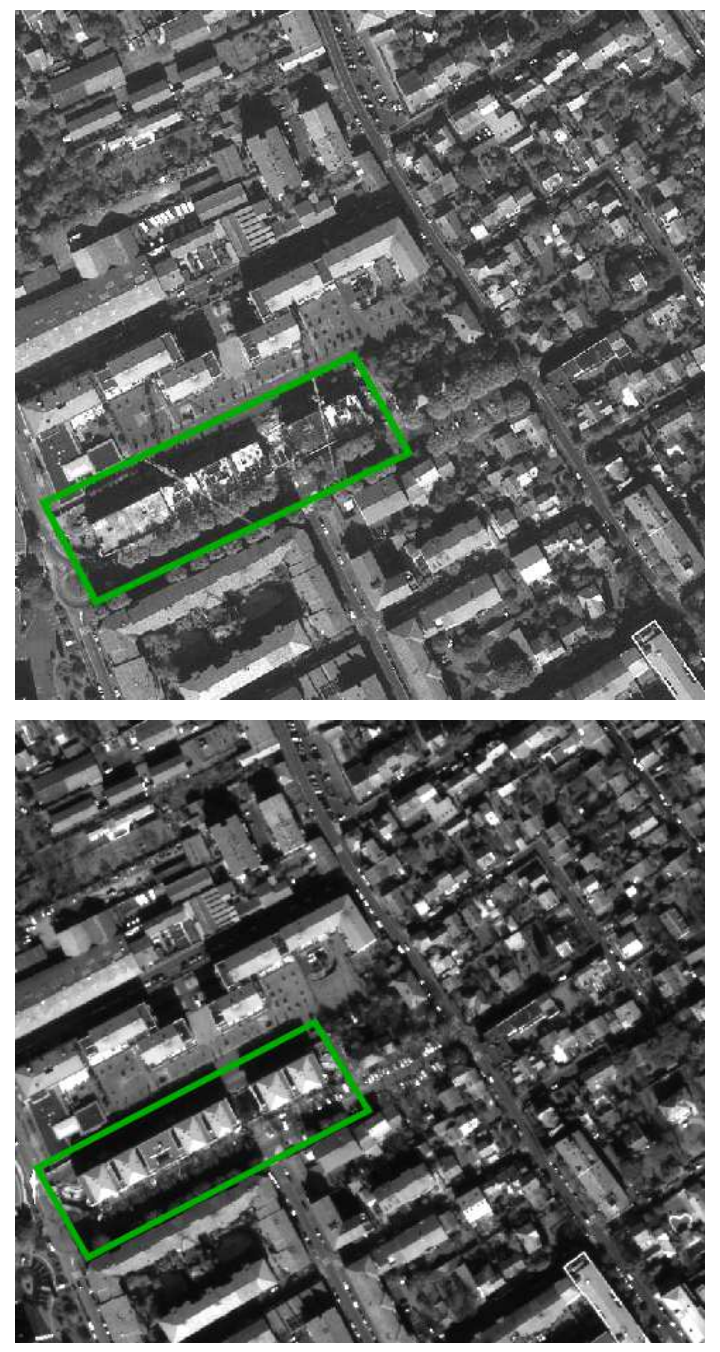

Fig. 1. Example of a pair of optical satellite images, with an apparent change surrounded in green (construction of a building). Both images have a resolution of $50 \mathrm{~cm}$ and an incidence angle close to nadir, but they have been acquired by different sensors (Geoeye and Worldview).

\subsection{Presentation of the SIFT algorithm}

The SIFT algorithm [1] follows three steps: first, interest points are detected as keypoints on two images. These keypoints are characterized by their position, scale and orientation. Then a descriptor is associated to each keypoint. These descriptors are designed to be invariant to translation, scaling and rotation. We use here a variant of the SIFT algorithm, presented in [5]. Keypoints from two images are finally matched by computing distances between their respective descriptors. To suppress false matches, we consider that images are roughly deformed by an affine transformation and we use an a contrario version of the RANSAC algorithm, called ACRANSAC [6]. We then obtain a set of matched keypoints. The SIFT algorithm has been designed for natural images,
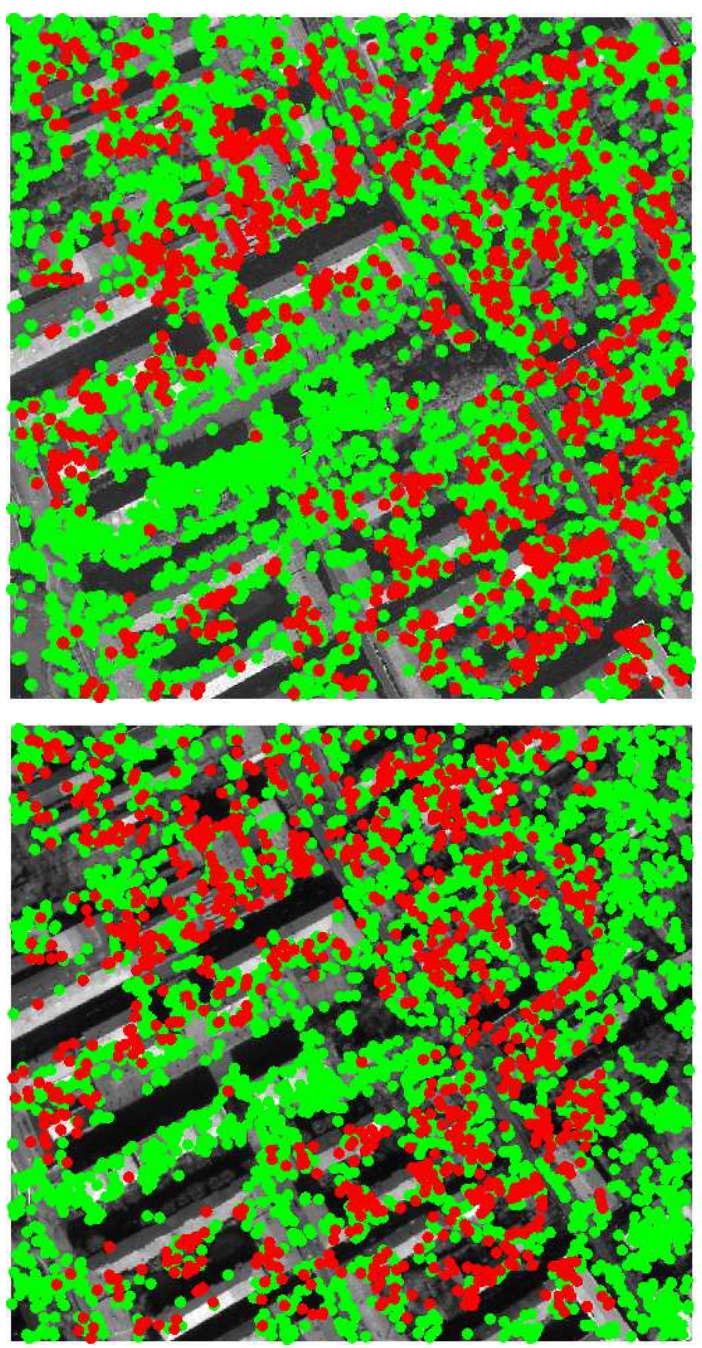

Fig. 2. Detected keypoints (in green) and matched keypoints (in red).

and works well on optical images. However it is not robust to the speckle noise of the SAR images. For these types of images, we use an adaptation of the SIFT algorithm, called SAR-SIFT [7].

\subsection{Local features and change detection situations}

Figure 2 presents the results of the SIFT algorithm applied to the two images of Figure 1: the green dots represent the detected keypoints and the red dots the matched keypoints, after AC-RANSAC filtering. Only the keypoints detected on objects common to the two images are considered. On the change areas, it can be observed that, while some keypoints are detected on both images, only a few are matched. On the areas presenting no apparent changes, the number of matched keypoints is much higher. Moreover the following observations can be made: in areas with no observed changes, the number of matched keypoints is weak compared to the num- 
ber of detected keypoints. Besides, the density of detected keypoints is not spatially uniform. In the following sections, we present an a contrario approach to locally compare the distributions of detected keypoints and matched keypoints respectively, in order to detect change areas.

\subsection{Principles of the a contrario approach}

The a contrario approach is based on the Helmholtz Principle [8]: human eyes notice a structure only if it unlikely appears randomly. This approach relies on two main concepts: a background model, that describes configurations where no structures should be detected ( $\mathcal{H}_{0}$ hypothesis), and a measurement $x$ on the structures to be detected. A significativity measure, called NFA (Number of False Alarms), is then assigned to each measurement, quantifying the unlikeliness of a given structure under the background model $\mathcal{H}_{0}$. The $N F A$ is obtained from the probability $P(X \geq x), X$ being the random variable that corresponds to the distribution of the measurement under the background model. Thresholding $N F A \leq \varepsilon$, with $\varepsilon$ small, allows us to detect structures.

\subsection{Change detection based on an a-contrario approach}

To detect changes, we make the following assumption: the density of detected keypoints, on an area $\mathcal{A}$, is the same as the density of matched keypoints. Knowing the density of matched keypoints on $\mathcal{A}$, we will be surprised if we find much more detected keypoints than expected. This situation describes well the change areas: we find, in proportion to the rest of the image, much more detected keypoints than matched keypoints.

To express this situation in an a contrario approach, we define the background model $\left(\mathcal{H}_{0}\right)$ as follows: the detected keypoints are distributed according to a Poisson process with locally the same density as the matched keypoints.

Given an area $\mathcal{A}$ of the image, the measurement $x$ is the number of detected keypoints actually found in $\mathcal{A}$, among the $N$ keypoints detected on the whole image. We define the variable $X$ as the distribution of the number of detected keypoints under hypothesis $\mathcal{H}_{0}$. We call $p$ the intensity of the Poisson process on the area $\mathcal{A}$. Considering that $N$ keypoints are detected on the image and that they are distributed according to a Poisson process, the variable $X$ follows a binomial law of parameter $p$. The probability of finding $x$ detected keypoints in $\mathcal{A}$ under hypothesis $\mathcal{H}_{0}$ is:

$$
P_{\mathcal{H}_{0}}(X=x)=\left(\begin{array}{c}
N \\
x
\end{array}\right) p^{x}(1-p)^{N-x}
$$

The $N F A$ is then obtained as the product of this probability by the number of detection tests performed on the image [8]:

$$
N F A=N \cdot P_{\mathcal{H}_{0}}(X \geq x)=N \cdot \sum_{i=x}^{N}\left(\begin{array}{c}
N \\
i
\end{array}\right) p^{i}(1-p)^{N-i}
$$
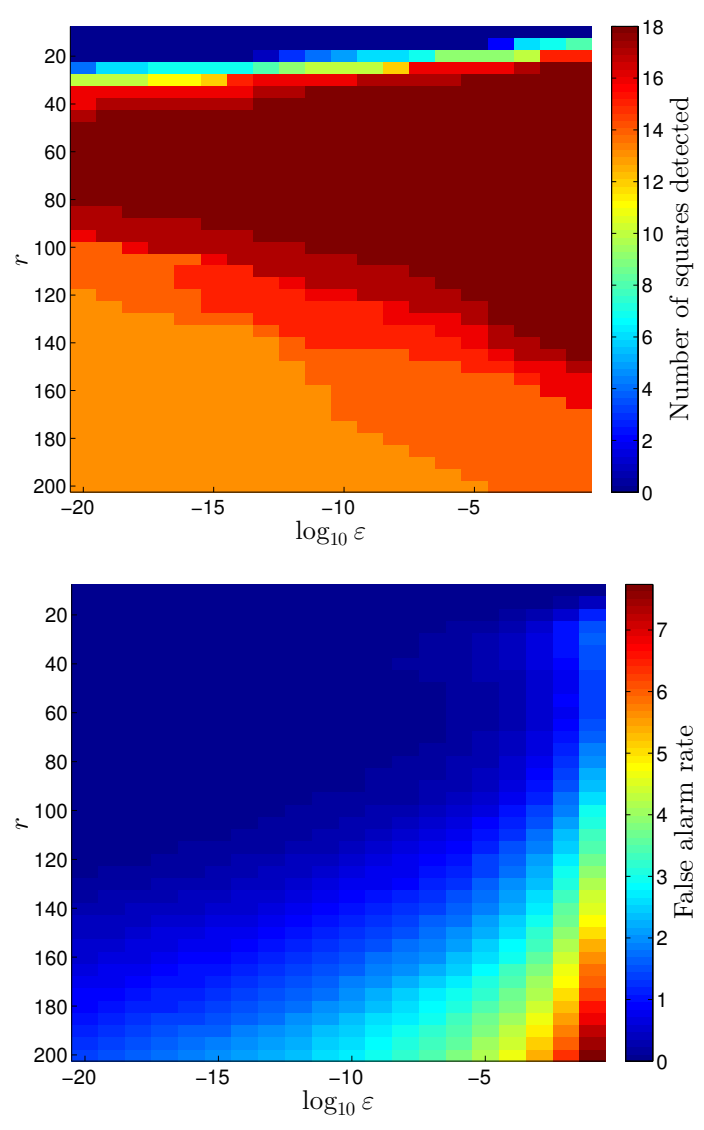

Fig. 3. Number of squares detected and false alarm rate for different values of $r$ and $\varepsilon$.

In practice, we define $\mathcal{A}$ as a circular neighborhood of radius $r$ centered around a keypoint. We define $n$ and $m$ respectively as the number of detected keypoints and matched keypoints in $\mathcal{A}$. We deduce the values $x=n$ and $p=\frac{m}{M}$, where $M$ is the number of matched keypoints on the whole image. We compute an $N F A$ for each keypoint. The keypoints are then classified as change with the help of a threshold $\varepsilon$ on $N F A$. The two parameters to set up are therefore the radius $r$ and the threshold $\varepsilon$.

\subsection{Results}

We first present a study of the influence of parameters, on simulated images. We have used optical and SAR denoised images, upon which we have added randomly some squares of homogeneous values and of different sizes. These squares represent the simulated changes. In total, nine squares have been added on each image, with size between 100 and 380 pixels. These sizes correspond roughly to changes observed on real images (construction and destruction of buildings). Some noise (gamma for the SAR image and gaussian for the optical image) has been added on each image in order to avoid identical keypoints and descriptors. We try to detect the 18 
changes, while having no false detections.

The proposed approach has been applied for different values of $r$ and $\varepsilon$ and two data are measured: the number of detection and the false alarm rate. The number of detection is defined as the number of squares upon which 30 keypoints are classified as change. Indeed, not all keypoints on the change areas need to be classified as change. It is only necessary that a sufficient number of keypoints are. The false alarm rate is computed as the percentage of keypoints wrongly classified as change. We can observe on Figure 3 the number of detection and the false alarm rate, with regards of $r$ and $\varepsilon$.

We can notice that the values of $r$ between 40 to 80 and with $\varepsilon$ lower than $10^{-10}$ should be considered. The number of detection is the highest, while presenting a false alarm rate close to zero.

Figure 4 presents a result for the two images of Figure 1. The parameters chosen here are $r=60$ (in pixel) and $\varepsilon=$ $10^{-15}$. We observe that the change area is properly detected on both images and there are no false detections. A detailed analysis of the performances of this method both for optical and SAR data and multiresolution images is currently under study.

\subsection{Conclusion}

A new change detection method for multi-sensors and multiresolutions situations has been presented. This algorithm relies on both the detections and matches of local features, combined with an a contrario approach. Further work includes a detailed analysis of performances and comparison with other approaches, as well as an automatic adaptation of the parameters.

\section{REFERENCES}

[1] D. G. Lowe, "Distinctive image features from scaleinvariant keypoints," International Journal of Computer Vision, 2004.

[2] O. Sjahputera, C.H. Davis, B.C. Claywell, N.J. Hudson, J.M. Keller, M. G. Vincent, Y. Li, M.N. Klaric, and C.-R. Shyu, "GeoCDX: An automated change detection and exploitation system for high resolution satelite imagery," in Geoscience and Remote Sensing Symposium (IGARSS), IEEE International, 2008.

[3] N. Bryant, W. Bunch, R. Fretz, P. Kim, T. Logan, M. Smyth, and A. Zobrist, "Obtaining accurate change detection results from high-resolution satellite sensors," in Applied Imagery Pattern Recognition Workshop (AIPR), 2012 IEEE, 2012.

[4] P. Gamba, F. Dell'Acqua, and G. Lisini, "Change detection of multitemporal SAR data in urban areas combining feature-based and pixel-based techniques," Geoscience and Remote Sensing, IEEE Transactions on, 2006.
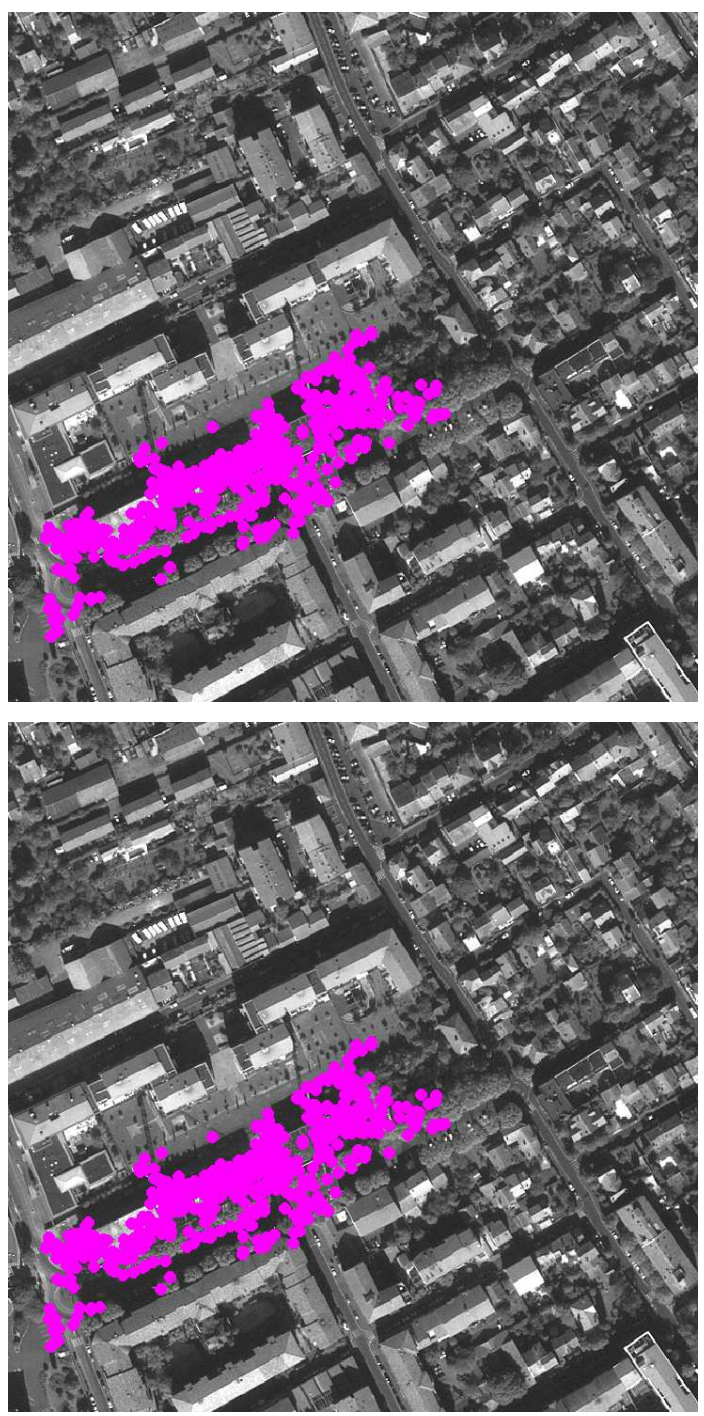

Fig. 4. Keypoints classified as change (example of Fig. 1).

[5] J. Rabin, J. Delon, and Y. Gousseau, "A statistical approach to the matching of local features," SIAM Journal on Imaging Sciences, 2009.

[6] J. Rabin, J. Delon, Y. Gousseau, and L. Moisan, "MACRANSAC: a robust algorithm for the recognition of multiple objects," in Proceedings of 3D Data Processing, Visualization and Transmission, 2010.

[7] F. Dellinger, J. Delon, Y. Gousseau, J. Michel, and F. Tupin, "SAR-SIFT: A SIFT-like algorithm for applications on SAR images," in IEEE International Geoscience and Remote Sensing Symposium, 2012.

[8] Agnes Desolneux, Lionel Moisan, and Jean-Michel Morel, From Gestalt Theory to Image Analysis: A Probabilistic Approach, Springer Publishing Company, Incorporated, 2007. 